JAMA. 2014 March 5; 311(9): 955-956. doi:10.1001/jama.2013.285478.

\title{
Acupuncture for chronic pain
}

\author{
Andrew J. Vickers, D.Phil and \\ Memorial Sloan-Kettering Cancer Center, New York, New York, USA \\ Klaus Linde, MD \\ Technical University, Munich, Germany
}

\section{Introduction}

Although acupuncture is widely used to manage chronic pain, it remains highly controversial, largely due to the lack of a clear mechanism. Several systematic reviews of acupuncture for chronic pain have been conducted. These have included trials of variable quality, typically leading a finding that weaknesses in the data do not allow conclusions to be drawn ${ }^{1}$. Meta-analyses have been limited due to variation in study endpoints used in randomized trials ${ }^{2}$. We conducted an individual patient data meta-analysis of acupuncture for chronic pain, restricted to high quality trials, with data available for 17,922 participants from 29 of 31 eligible trials ${ }^{3}$.

\section{Summary of findings}

The 29 trials included 18 comparisons of acupuncture vs. no acupuncture control (typically routine care; $n=14,597$ ) and 20 comparisons of acupuncture with sham acupuncture $(n=5,230)$. Four sham-controlled trials were determined to have an intermediate likelihood of bias from unblinding. The 16 remaining sham-controlled trials were graded as having a low risk of bias from unblinding.

Analyses were conducted separately according to the comparison group (no acupuncture control or sham acupuncture) and pain condition (non-specific musculoskeletal pain, osteoarthritis, chronic headache, shoulder pain). Acupuncture was associated with greater reductions in pain than control in all comparisons $(\mathrm{p}<0.001)$. The test for heterogeneity was statistically significant for all comparisons with sham, and for the no acupuncture comparisons for musculoskeletal pain and osteoarthritis. In the case of comparisons with sham acupuncture, three trials by one team were outliers with much larger effect sizes than average. After exclusion of these trials, there was no significant heterogeneity in the comparisons between acupuncture and sham, the effect size for acupuncture vs. sham was lower for osteoarthritis and musculoskeletal pain and effect sizes became relatively similar for the different pain conditions. For back and neck pain, osteoarthritis, and chronic headache respectively, pain scores were $0.23,0.16$ and 0.15 standard deviations better for

Corresponding Author: Andrew Vickers, Department of Epidemiology \& Biostatistics, Memorial Sloan-Kettering Cancer Center, 307 East 63rd Street, 2nd floor NY, NY 10021, t: 646.735.8142, f: 646.735.0011, vickersa@ mskcc.org.

Conflicts of Interest: All authors report no conflicts of interest. 
acupuncture compared to sham, and $0.55,0.57$ and 0.42 standard deviations better when compared against no acupuncture control.

To translate these results into clinical terms, we defined a good response in terms of a pain reduction of $50 \%$ or more and applied the differences between groups from the metaanalysis to a hypothetical trial with a typical mean and standard deviation for pain score. Response rates of approximately $30 \%, 42.5 \%$ and $50 \%$ for no treatment, sham acupuncture and real acupuncture respectively were calculated by dividing means by standard deviation and then applying to a normal distribution. These results were robust to a number of sensitivity analyses, including those for missing data, publication bias, inclusion of trials for which individual patient data were not available (including all eligible trials published to 2010), exclusion of four trials for which blinding was unclear (for back and neck pain, effect size 0.01 lower at $0.36,95 \%$ C.I. 0.25 to 0.46 , p $<0.001$; for chronic headache, effect size 0.01 lower at $0.14,95 \%$ C.I. 0.03 to $0.25, \mathrm{p}=0.01$ ), and restricting endpoints to pain scales measured within 2 to 3 months of randomization.

\section{Comment}

Acupuncture is associated with reductions in chronic pain as compared to sham acupuncture and as compared to no acupuncture control. Differences between true and sham acupuncture are smaller than those between true acupuncture and no acupuncture control. The search for eligible trials was repeated in October 2013. A table of eligible papers published 2011 2013 is included in an online appendix. There is no reason to believe that recently published data would change the results of the meta-analysis as results are either very similar to the meta-analytic estimates or the trials very small.

\section{Limitations}

Participants were not blinded to the comparison between acupuncture and no acupuncture control and therefore may be subject to bias. The number of trials on shoulder pain was limited, and the effect size of acupuncture for this indication may not have been wellcharacterized by our data.

\section{Comparison of Findings with Current Guidelines}

Many current guidelines recommend acupuncture in specific circumstances for back pain or headache. For instance, the American College of Physicians' guidelines recommend acupuncture as one of several options, such as manual therapy or exercise, for patients with back pain ${ }^{4}$; the UK National Institute for Health and Care Excellence (NICE) guidelines recommend acupuncture for chronic headache or migraine ${ }^{5}$ that does not respond to pharmacologic treatment. However, the evidence in favor of these guidelines has been reported as "fair". There have been recommendations against using acupuncture for osteoarthritis in several guidelines including those from $\mathrm{NICE}^{6}$ and the American Academy of Orthopedic Surgeons ${ }^{7}$. No current guidelines recommend acupuncture for neck or shoulder pain. 


\section{Areas in Need of Future Study}

The sham acupuncture techniques varied and included several control conditions that involved skin penetration. It is remains to be established whether this type of sham is indeed physiologically inactive: if not, trials that include sham acupuncture as a comparison may underestimate the effects of acupuncture on pain reduction. Research is also needed to better identify which patients will benefit most from acupuncture, and where in the normal stepped-care approaches for chronic pain acupuncture is best positioned.

\section{Evidence Profile}

No. of studies: 31 of which 29 had individual patient data available for inclusion in the primary analysis.

No. of randomized controlled trials: 31 of which 11 were sham-controlled, 10 had a no acupuncture control and 10 were 3 -armed studies including both sham and no-acupuncture control.

Data collection: 1996 - 2008.

No. of patients: 14,597 for studies in which a no acupuncture control was used; 5,230 for studies in which the control was sham acupuncture

Male: Female: 5624 (30.5\%) $12535(68 \%)$. Missing data on 275 (1.5\%)

Race/ethnicity: Unavailable

Age: Median: 51; quartiles 39, 63; range $17-95$.

Settings: Both general practices in the community and hospital based settings.

Countries: US, UK, Germany, Spain, Sweden

Comparisons: Acupuncture vs. no acupuncture control and acupuncture vs. sham acupuncture.

Primary Outcome: A variety of different pain and function scores were used in the original trials; these were converted to standardized differences in the meta-analysis

Secondary Outcomes: Not applicable

\section{Supplementary Material}

Refer to Web version on PubMed Central for supplementary material.

\section{Acknowledgments}

Funding Support: Funded by an R21 and R01 (AT004189I and R01 AT006794) from the National Center for Complementary and Alternative Medicine (NCCAM) at the National Institutes of Health (NIH) to Dr Vickers and by a grant from the Samueli Institute. 
Role of the Sponsor: AV had full access to all of the data in the study and takes responsibility for the integrity of the data and the accuracy of the data analysis. The sponsors had no role in study design, data collection and analysis, decision to publish, or preparation of the manuscript.

\section{References}

1. Furlan AD, van Tulder MW, Cherkin DC, et al. Acupuncture and dry-needling for low back pain. The Cochrane database of systematic reviews. 2005; (1):CD001351. [PubMed: 15674876]

2. Ezzo J, Hadhazy V, Birch S, et al. Acupuncture for osteoarthritis of the knee: a systematic review. Arthritis and rheumatism. Apr; 2001 44(4):819-825. [PubMed: 11315921]

3. Vickers AJ, Cronin AM, Maschino AC, et al. Acupuncture for chronic pain: individual patient data meta-analysis. Archives of internal medicine. Oct 22; 2012 172(19):1444-1453. [PubMed: 22965186]

4. Chou R, Qaseem A, Snow V, et al. Diagnosis and treatment of low back pain: a joint clinical practice guideline from the American College of Physicians and the American Pain Society. Annals of internal medicine. Oct 2; 2007 147(7):478-491. [PubMed: 17909209]

5. National Institute for Health and Clinical Excellence. Headaches: Diagnosis and management of headaches in young people and adults. 2012. http://www.nice.org.uk/nicemedia/live/ 13901/60853/60853.pdf

6. Conaghan PG, Dickson J, Grant RL. Care and management of osteoarthritis in adults: summary of NICE guidance. BMJ (Clinical research ed.). Mar 1; 2008 336(7642):502-503.

7. American Academy of Orthopaedic Surgeons. Evidence Based Guideline. 2nd Edition. 2013. Treatment of Osteoarthritis of the Knee. http://www.aaos.org/research/guidelines/ TreatmentofOsteoarthritisoftheKneeGuideline.pdf 


\section{Clinical question}

Is acupuncture associated with reduced pain outcomes for patients with chronic pain as compared to sham ("placebo") acupuncture or no acupuncture control, respectively? 


\section{Bottom line}

Acupuncture is associated with improved pain outcomes compared to sham acupuncture and compared to no acupuncture control, with response rates of approximately $30 \%$, $42.5 \%$ and $50 \%$ for no acupuncture, sham acupuncture and real acupuncture respectively 


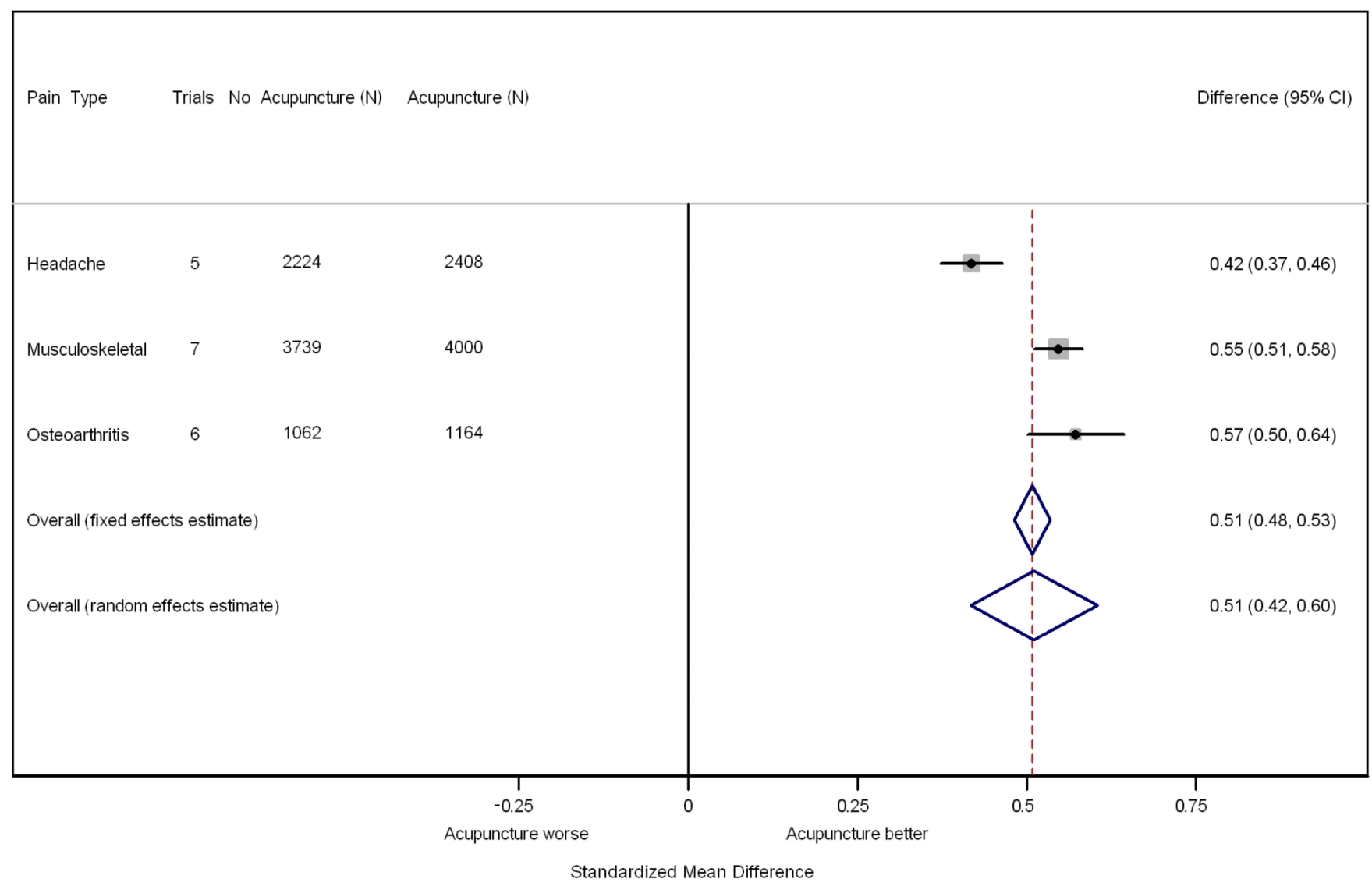

Fig 1.

Results of the individual patient data meta-analysis of acupuncture versus sham and versus no acupuncture, as standardized mean difference. A standardized mean difference of 0.42 , for instance, means that pain scores were 0.42 standard deviations lower in patients undergoing acupuncture compared to controls. These differences are converted to clinically meaningful statistics in the text. 


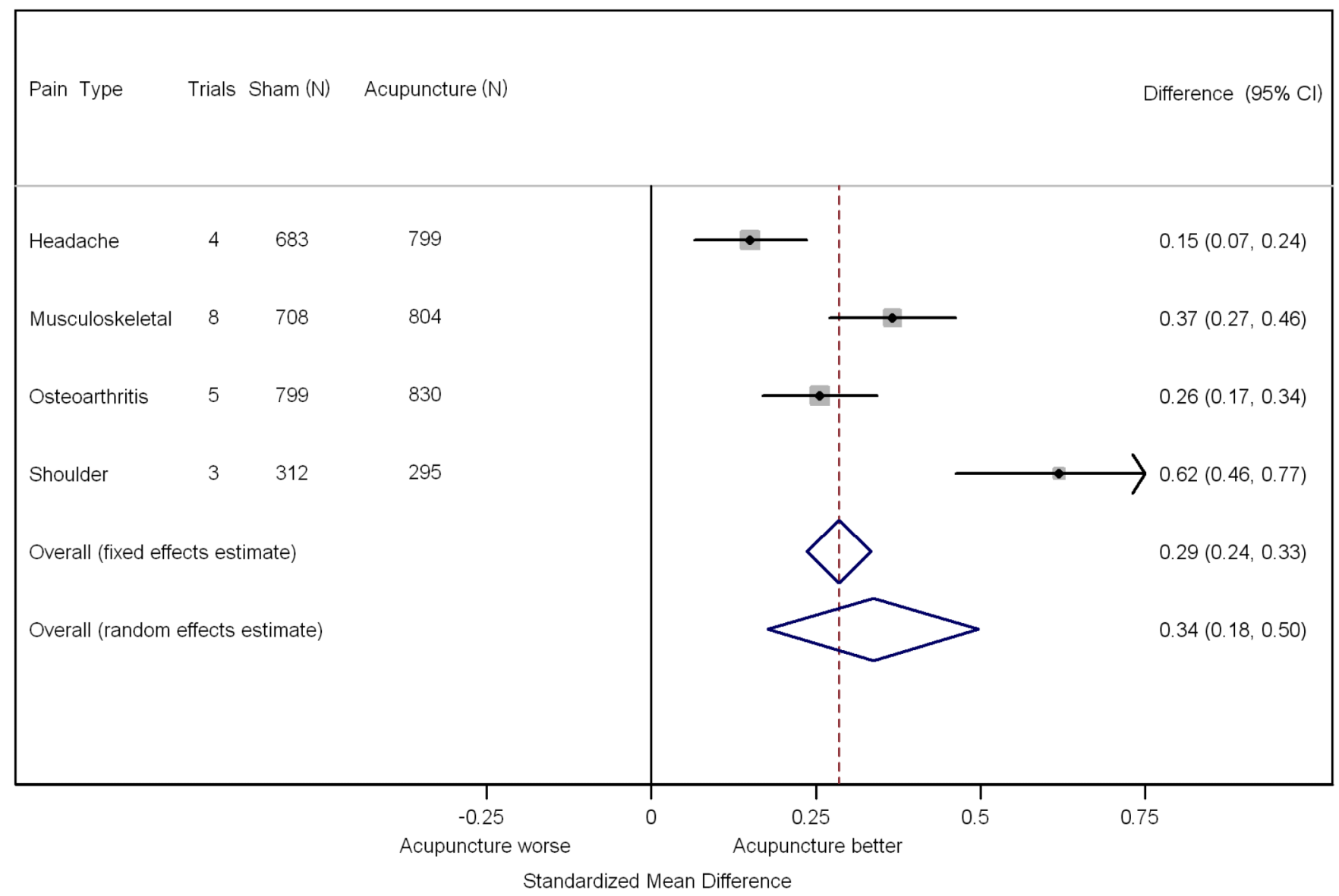

Fig 2. 